\title{
A European survey of management approaches in chronic urticaria in children: EAACI Paediatric Urticaria Taskforce
}

\author{
Sophia Tsabouri ${ }^{1}$, Stefania Arasi ${ }^{2}$, Burcin Beken ${ }^{3}$, Martin Church $^{4}$, Montserrat Alvaro $^{5}$, \\ Carlo Caffarelli ${ }^{6}$, Carsten Flohr ${ }^{7}$, Sherief Janmohamed ${ }^{8}$, George Konstantinou ${ }^{9}$, Susanne \\ Lau $^{10}$, Sebastian Lefevre ${ }^{11}$, Charlotte Mørtz ${ }^{12}$, Giovanni Pajno ${ }^{13}$, Helena Pite ${ }^{14}$, Chris \\ Rutkowski ${ }^{7}$, Petra Staubach ${ }^{15}$, Lauri Ann van der Poel ${ }^{16}$, Torsten Zuberbier ${ }^{17}$, and Tabie \\ Anika Leslie ${ }^{18}$ \\ ${ }^{1}$ University of Ioannina Faculty of Medicine \\ ${ }^{2}$ Ospedale Pediatrico Bambino Gesu \\ ${ }^{3}$ Trakya Universitesi \\ ${ }^{4}$ Charité, Universitätsmedizin Berlin \\ ${ }^{5}$ Hospital Sant Joan de Deu \\ ${ }^{6}$ Università di Parma \\ ${ }^{7}$ Guy's and St Thomas' NHS Foundation Trust \\ ${ }^{8}$ Universitair Ziekenhuis Brussel \\ ${ }^{9} 424$ General Military Training Hospital \\ ${ }^{10}$ Charité Campus Virchow \\ ${ }^{11}$ Regional Hospital Centre Metz-Thionville \\ ${ }^{12}$ Odense University Hospital \\ ${ }^{13}$ University of Messina \\ ${ }^{14}$ Centro Hospitalar Lisboa Central \\ ${ }^{15}$ University Medical Center of Mainz, Johannes Gutenberg-University Mainz \\ ${ }^{16}$ Guy's and Saint Thomas' NHS Foundation Trust \\ ${ }^{17}$ Charité - Universitätsmedizin Berlin \\ ${ }^{18}$ Royal Free Hospital
}

September 24, 2021

\begin{abstract}
Background: Although well described in adults, there are scarce and heterogeneous data on the diagnosis and management of chronic urticaria (CU) in children (0-18 years) throughout Europe. Our aim was to explore country differences and identify the extent to which the EAACI/GA ${ }^{2} \mathrm{LEN} / \mathrm{EDF} / \mathrm{WAO}$ guideline recommendations for paediatric urticaria are implemented. Methods: The EAACI Taskforce for paediatric CU disseminated an online clinical survey among EAACI paediatric section members. Members were asked to answer 35 multiple choice questions on current practices in their respective centres. Results: The survey was sent to 2,773 physicians of whom 358 (13.8\%) responded, mainly paediatric allergists (80\%) and paediatricians (49.7\%), working in 69 countries. For diagnosis, Southern European countries used significantly more routine tests (e.g., autoimmune testing, allergological tests, and parasitic investigation) than Northern European countries. Most respondents (60.3\%) used a 2nd generation antihistamine as first- line treatment of whom $64.8 \%$ up dosed as a second- line. Omalizumab, was used as a second line treatment by $1.7 \%$ and third-line by $20.7 \%$ of respondents. Most clinicians $(65 \%)$ follow EAACI/WAO/GA2LEN/EDF guidelines when diagnosing CU, and only $7.3 \%$ follow no specific guidelines. Some clinicians prefer to follow national guidelines
\end{abstract}


(18.4\%, mainly Northern European) or the AAAAI practice parameter (1.7\%). Conclusions: Even though most members of the Paediatric Section of EAACI are familiar with the EAACI/WAO/GA2LEN/EDF guidelines, a significant number do not follow them. Also, the large variation in diagnosis and treatment strengthens the need to re-evaluate, update and standardize guidelines on the diagnosis and management of $\mathrm{CU}$ in children.

\section{Hosted file}

A European survey of management approaches in chronic urticaria in children EAACI Paediatric Urticaria " available at https://authorea.com/users/351635/articles/538502-a-european-survey-ofmanagement-approaches-in-chronic-urticaria-in-children-eaaci-paediatric-urticariataskforce

\section{Figure 1. Percentage of chronic urticaria patients complain} of angioedema as indicated by the respondents
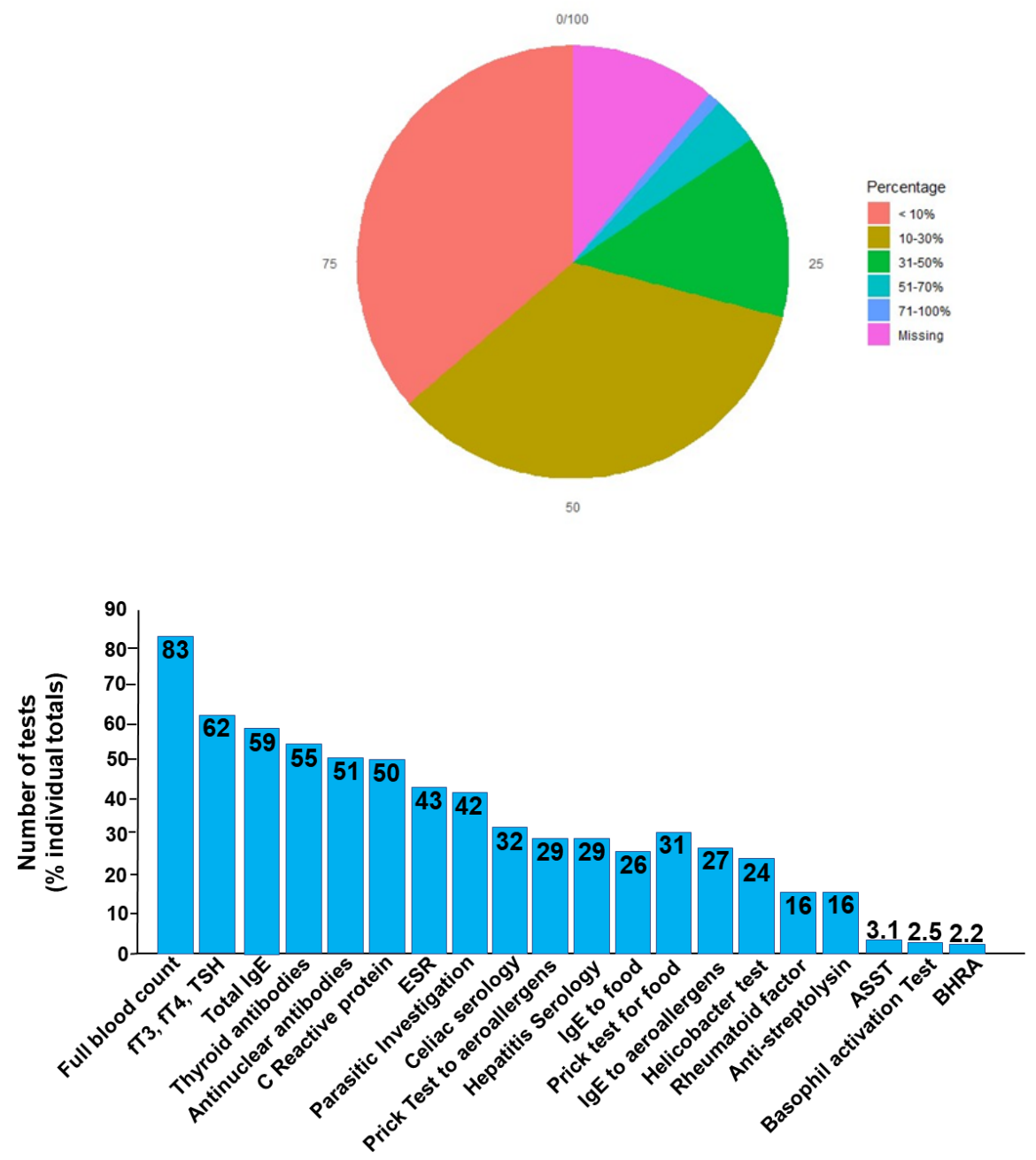


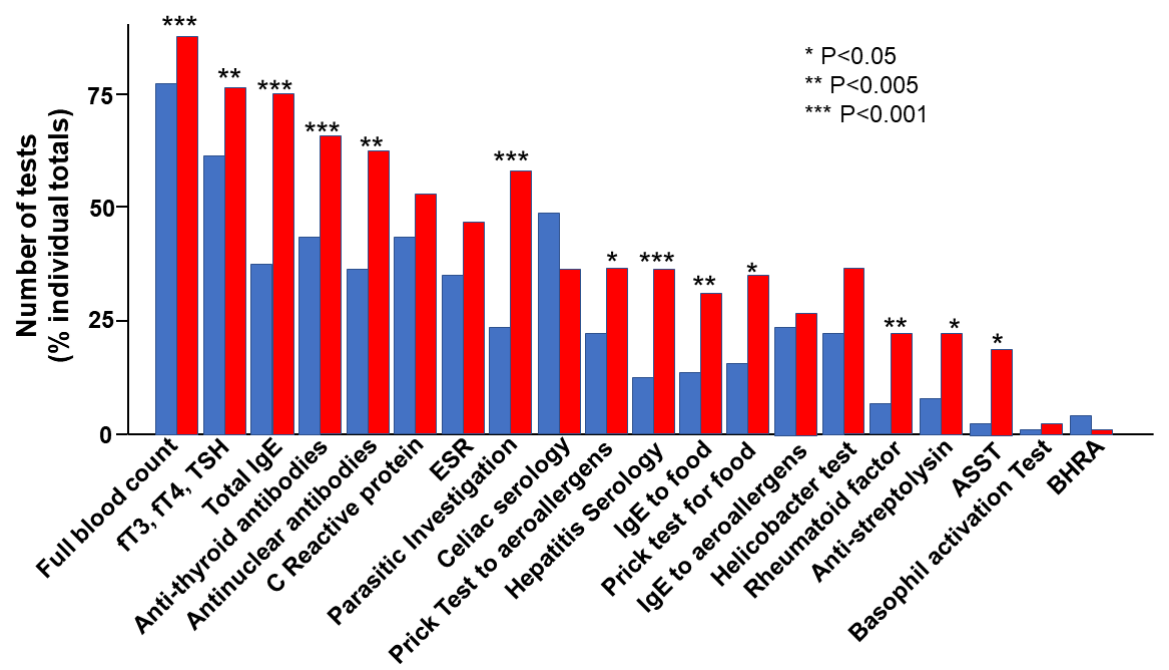

